\title{
INEQUALITIES FOR THE POWERS OF NONNEGATIVE HERMITIAN OPERATORS
}

\section{MAN KAM KWONG}

ABSTRACT. In the set of bounded Hermitian operators from a Hilbert space $H$ into itself, we define three types of ordering by means of the cones of nonnegative, positive definite and positive invertible operators respectively. Our main theorem shows that for all three types of ordering, if $A$ is "greater" than $B$, then $A^{r}$ is "greater" than $B^{r}$ for all real numbers $r \leq 1$. This generalizes the results of Heinz [3] and Kato [4].

1. Introduction and statement of the theorem. Let $H$ be a Hilbert space over the complex numbers, with inner product $\langle\cdot, \cdot\rangle$ and norm $\|\cdot\|$, and $A$ be the set of bounded nonnegative Hermitian linear operators from $H$ into itself. We shall distinguish three types of ordering in $\mathbf{A}$. Let $A, B \in \mathbf{A}$, and $H^{*}=$ $\{u \in H: u \neq 0\}$.

(1) $A \geq B$ iff $\langle u, A u\rangle \geq\langle u, B u\rangle$ for all $u \in H$.

(2) $A\rangle B$ iff $\langle u, A u\rangle\rangle\langle u, B u\rangle$ for all $u \in H^{*}$.

(3) $A \gg B$ iff $A-B>0$ and is invertible.

We shall use the symbol $>$ when we mean any one of the three types of ordering. Our main purpose is to prove

Theorem 1. If $A, B \in \mathrm{A}$ and $A>B$, then $A^{r}>B^{r}$ for any $0<r \leq 1$.

In the case when $H$ is finite dimensional, the theorem is a well-known result of Loewner's [5] concerning matrices. Au-Yeung recently gave a simple proof in [1].

In the infinite-dimensional case, the same theorem for the ordering $\geq$ was first given by Heinz [3, Satz 3]. Kato [4] gave an elementary proof. In this paper, we give another elementary proof as well as a generalization of the result to the orderings $>$ and $\gg$. Moreover, the classical theorem of Heinz is strengthened as stated in Theorem 2 of $\$ 5$. The author wishes to thank Dr. Y. H. Au-Yeung for suggesting this problem and for his inspiring discussions.

Notice that in finite-dimensional spaces, (2) and (3) are equivalent.

Let $\mathrm{A}^{*}=\{A \in \mathrm{A}: A \gg 0\}$. Then $A \in \mathrm{A}^{*}$ iff there is a positive real num-

Received by the editors September 4, 1973 and, in revised form, June 10, 1974. AMS (MOS) subject classifications (1970). Primary 47B15. 
ber $m$ such that $A \geq m I$ 。 Here $I$ denotes the identity mapping.

We point out the following simple but useful fact.

Lemma l. If $A, B \in \mathbf{A}$ and $A>B$, then for every $D \in \mathbf{A}^{*}, D A D>D B D$. Conversely, if there exists a $D \in \mathrm{A}^{*}$ such that $D A D>D B D$, then $A>B$.

2. The special case $r=1 / 2$, We need the following lemmas.

Lemma 2. Suppose that $A \in \mathrm{A}^{*}, B \in \mathrm{A}$ and $\alpha$ is the supremum of the quotients $\|B u\| /\|A u\|$ for all $u \in H$ and $u \neq 0$. Then $\langle u, B u\rangle \leq a<u, A u\rangle$ for all $u \in H$.

Proof. $A \in \mathrm{A}^{*}$ implies that $A^{1 / 2} \in \mathrm{A}^{*}$, and so $D=A^{-1 / 2} \in \mathrm{A}^{*}$. Let $C=$ $D B D \in \mathrm{A}$. By the well-known theory of integral representation of Hermitian operators, there is a spectral decomposition associated with $C$ (see, for example, [2, p. 275]) say $\left\{E_{\lambda}\right\}$ for $-\infty<\lambda<\alpha$, and $C=\int_{0-}^{\beta} \lambda d E_{\lambda}$ where $\beta=$ $\|C\|$. Hence $\langle u, C u\rangle=\int_{0-}^{\beta} \lambda d\left\|E_{\lambda}\right\|^{2}$ from which we obtain $\sup \langle\langle u, C u\rangle$ : $u \in H,\|u\|=1\}=\beta$. It is not difficult to see that

$$
\sup \{\langle u, B u\rangle /\langle u, A u\rangle: u \in H, u \neq 0\}=\sup \{\langle u, C u\rangle: u \in H,\|u\|=1\} .
$$

The lemma will be proved if given any $\epsilon>0$, we can exhibit a $v \epsilon H$ such that $\|B v\| /\|A v\| \geq \beta-\epsilon_{\text {. }}$ Let

$$
\theta=\epsilon\left\|A^{-1}\right\| / 2\left\|A^{1 / 2}\right\|^{2},
$$

and $\lambda_{0}=\beta-\theta$. When $\epsilon$ is small enough, $\lambda_{0}>0$. Choose a nonzero vector $u \in H$ such that $E_{\lambda} u=0$ for some $\lambda$ such that $\lambda_{0}<\lambda<\beta$. Finally define $v=D u$. Since

$$
B v=A^{1 / 2} \int_{\lambda_{0}}^{\beta} \lambda d E_{\lambda}\left(A^{1 / 2} v\right)
$$

and

$$
A v=A^{1 / 2} u=A^{1 / 2} \int_{\lambda_{0}}^{\beta} d E_{\lambda}\left(A^{1 / 2} v\right)
$$

we have

$$
\begin{aligned}
\left\|B v-\lambda_{0} A v\right\| & =\left\|A^{1 / 2} \int_{\lambda_{0}}^{\beta}\left(\lambda-\lambda_{0}\right) d E_{\lambda}\left(A^{1 / 2} v\right)\right\| \\
& \leq\left\|A^{1 / 2}\right\| \theta\left\|A^{1 / 2} v\right\| \leq \theta\left\|A^{1 / 2}\right\|^{2}\|v\| .
\end{aligned}
$$

Hence

$$
\begin{aligned}
\|B v\| /\|A v\| & \geq\left(\left\|\lambda_{0} A v\right\|-\left\|B v-\lambda_{0} A v\right\|\right) /\|A v\| \\
& \geq \lambda_{0}-\left(\theta\left\|A^{1 / 2}\right\|^{2}\|v\|\right) /\left\|A^{-1}\right\| \cdot\|v\|=\beta-\epsilon .
\end{aligned}
$$


Lemma 3. Suppose that $A, B \in \mathrm{A}$ and $A \geq B$. If $u_{0}$ is a vector in $H^{*}$ such that $\left\langle u_{0}, B u_{0}\right\rangle=\left\langle u_{0}, A u_{0}\right\rangle$, then $A u_{0}=B u_{0}$. In other words, $A \ngtr B$ implies that $A^{2} \ngtr B^{2}$.

This is a well-known result. See, for example $[6$, p. 327 , equation $(6.11$ $-7)]$.

Lemma 4. $A, B \in \mathbf{A}$ and $A^{2} \geq B^{2}$, then $A \geq B$.

Proof. First assume that $A \in \mathrm{A}^{*}$. Then from hypothesis, $\left\langle u, A^{2} u\right\rangle \geq$ $<u, B^{2} u>$ for all $u \in H$. Thus $\|B u\| /\|A u\| \leq 1$ for all $u \in H, u \neq 0$. By Lemma 2,

$$
\langle u, B u\rangle /\langle u, A u\rangle \leq \alpha \leq 1
$$

This is the required conclusion. In general, $A+n I \in \mathrm{A}^{*}$ for all positive $n$ and $(A+n I)^{2}=A^{2}+2 n A+n^{2} I \geq B^{2}$. Thus $A+n I \geq B$. Let $n$ go to zero.

Lemma 5. $A, B \in \mathbf{A}$ and $A^{2}>B^{2}$, then $A>B$.

Proof. By Lemma $4, A \geq B$. Then apply Lemma 3 .

Lemma 6. $A, B \in \mathrm{A}$ and $A^{2} \gg B^{2}$, then $A \gg B$.

Proof. For some positive $m, A^{2} \geq B^{2}+m I$. As $B$ is bounded, $\|B\| I \geq B$. Choose $n$ so small that $\left(m-n^{2}\right) I \geq 2 n B$. Then $A^{2} \geq B^{2}+m I \geq B^{2}+2 n B+$ $n^{2}$ l. By Lemma $4, A \geq B+n I$.

3. Some more inequalities.

Lemma 7. Let $X, Y \in \mathrm{A}$ and $C, D \in \mathrm{A}^{*}$ such that $C \geq D$. If $Y D Y>$ $X C X$, then $Y>X$.

Proof. By Lemma 1, YCY $\geq Y D Y$. Thus $Y C Y>X C X$. So

$$
\left(C^{1 / 2} Y C^{1 / 2}\right)\left(C^{1 / 2} Y C^{1 / 2}\right)>\left(C^{1 / 2} X C^{1 / 2}\right)\left(C^{1 / 2} X C^{1 / 2}\right) \text {. }
$$

By Lemmas 4, 5 and 6, $C^{1 / 2} Y C^{1 / 2}>C^{1 / 2} X C^{1 / 2}$. Lemma 1 then gives the conclusion.

Lemma 8. If $A \in \mathrm{A}^{*}, B \in \mathrm{A}$ and $A^{r} \geq B^{r}$, then

$$
B^{s} \geq B^{(r+s) / 2} A^{-r} B^{(r+s) / 2}
$$

where $r, s$ are real numbers.

Proof. Notice two simple facts. First, if $X \in \mathrm{A}^{*}$ and $X>I$, then $I>$ $X^{-1}$ (by Lemma 1 using $X, I$ and $X^{-1 / 2}$ in place of $A, B$ and $D$ ). Second, if $X, Y \in \mathrm{A}^{*}$ and $Y>X$, then $X^{-1}>Y^{-1}$ (by Lemma $1, X^{-1 / 2} Y X^{-1 / 2}>I$, 
so $I>\left(X^{-1 / 2} Y X^{-1 / 2}\right)^{-1}=X^{1 / 2} Y^{-1} X^{1 / 2} ;$ then apply Lemma 1 again $)$. Now assume that $A, B \in \mathrm{A}^{*}$ and $A^{r} \geq B^{r}$. Then $B^{-r} \geq A^{-r}$. Lemma 1 then gives the conclusion. In general, consider $A^{r}+n I$ and $B^{r}+n I$ for any positive $n$. The above implies that

$$
\left(B^{r}+n I\right)^{s / r} \geq\left(B^{r}+n I\right)^{(s+r) / 2 r}\left(A^{r}+n I\right)^{-1}\left(B^{r}+n l\right)^{(s+r) / 2 r} .
$$

Let $n$ tend to zero.

Lemma 9. If $A \in \mathrm{A}^{*}, B \in \mathrm{A}$ and if $r, s$ are two real numbers such that $A^{r} \geq B^{r}, A^{s}>B^{s}$, then $A^{(r+s) / 2}>B^{(r+s) / 2}$.

Proof. $A^{(r+s) / 2} A^{-r} A^{(r+s) / 2}=A^{s}>B^{s} \geq B^{(r+s) / 2} A^{-r} B^{(r+s) / 2}$. Then use Lemma 7.

4. Proof of the main theorem $(\geq, \gg)$. First assume that $A \in \mathrm{A}^{*}$. This is no restriction in case $A \gg B$. Then Lemmas $4,5,6$ and 9 show that $A^{q}>$ $B^{q}$ if $q$ is any dyadic fraction in $[0,1]$. The set of dyadic fractions is dense in $[0,1]$. A continuity argument lifts the restriction that $A \in \mathrm{A}^{*}$, for the case $A \geq B$. When $A \gg B$, we have $A \geq B+m I$ for some positive $m$. Thus $A^{r} \geq$ $(B+m I)^{r}$. The function $\left[(\lambda+m)^{r}-\lambda^{r}\right]$ being positive on the closed interval $[0,\|B\|]$ has a positive lower bound $n$. Then using the spectral decomposition, we see without difficulty that $(B+m I)^{r} \geq B^{r}+n I$. Hence $A^{r} \gg B^{r}$. The case where $A>B$ needs a little more effort and will be proved in the next section.

5. Proof of the main theorem $(>)$.

Lemma 10. Suppose that $A, B \in \mathrm{A}$ and $A^{2} \geq B^{2}$. Furthermore there exists a vector $u_{0} \in H^{*}$ such that $A u_{0}=B u_{0}$. Then $F(A) u_{0}=F(B) u_{0}$ for any continuous function $F$.

Proof. Let $E=\{u \in H: A u=B u\}$. By Lemma $3, A^{2} u=B^{2} u$ for all $u \in E$. In particular, $A^{2} u_{0}=B^{2} u_{0}=B A u_{0}$. Hence $A u_{0}$ again belongs to $E$. Thus $A^{3} u_{0}=B^{2} A u_{0}=B^{3} u_{0}$. By induction, $A^{n} u_{0}=B^{n} u_{0}$ for any positive integer $n$. By Weierstrass approximation theorem, $F$ can be uniformly approximated by polynomials, and hence the conclusion.

Corollary l. If $A, B \in \mathrm{A}$ and $A \geq B$, then unless there exists a $u_{0} \in H^{*}$ such that $F(A) u_{0}=F(B) u_{0}$ for all continuous functions $F$, we have $A^{r}>B^{r}$ for all $r<1 / 2$.

Corollary 2. $A, B \in \mathrm{A}\left(A \in \mathrm{A}^{*}\right)$ and $A \geq B$. Then, unless there exists a $u_{0} \in H^{*}$ such that $F(A) u_{0}=F(B) u_{0}$ for all continuous functions $F, A^{r}>B^{r}$ for all $r<1$. 
Proof. Suppose that there is an $r<1$ such that $A^{r} \ngtr B^{r}$. By Corollary 1 , $r>1 / 2$. So $\theta=1-r<1 / 2$. By Lemma 9, $A^{1-2 \theta} \ngtr B^{1-2 \theta}$. We can continue the process for $n$ steps until we have $2^{n} \theta>1 / 2$. This contradicts Corollary 1 . Notice that the restriction $A \in \mathrm{A}^{*}$ can be removed after we prove Lemma 11 .

Lemma 11. Lemma 9 in the case $A^{s}>B^{s}$ is still true even if $A \in \mathbf{A}$ only.

Proof. From the main theorem for the ordering $\geq$, we have $A^{(r+s) / 2} \geq$ $B^{(r+s) / 2}$, so $A^{(r+s) / 2}+I \geq B^{(r+s) / 2}+I$. Thus $\left(A^{(r+s) / 2}+I\right)^{\theta} \geq\left(B^{(r+s) / 2}+I\right)^{\theta}$ for all $\theta \leq 1$. Choose $\theta$ so small that $(r+s)(1+\theta) / 2 \leq r$. We study the functions

$$
f(\alpha, u)=\left\langle u,\left(A^{(r+s) / 2}+\alpha\right)^{1+\theta} u\right\rangle, \quad g(\alpha, u)=\left\langle u,\left(B^{(r+s) / 2}+\alpha\right)^{1+\theta} u\right\rangle
$$

for $u \in H, a$ real and $\geq 0$.

Now

$$
d f(\alpha, u) / d \alpha=(1+\theta)\left\langle u,\left(A^{(r+s) / 2}+\alpha\right)^{\theta} u\right\rangle .
$$

Thus

$$
d f(\alpha, u) / d \alpha \geq d g(\alpha, u) / d \alpha .
$$

This implies that

$$
f(1, u)=f(0, u)+\int_{0}^{1} \frac{d f}{d \alpha} d a \geq g(0, u)+\int_{0}^{1} \frac{d g}{d a} d \alpha=g(1, u) .
$$

Here $f(0, u) \geq g(0, u)$. Thus $\left(A^{(r+s) / 2}+I\right)^{1+\theta} \geq\left(B^{(r+s) / 2}+I\right)^{1+\theta}$. By Corollary $2,\left(A^{(r+s) / 2}+I\right)>\left(B^{(r+s) / 2}+I\right)$, unless there exists a $u_{0} \in H^{*}$, having the property stated there, in particular $A^{s} u_{0}=B^{s} u_{0}$, contradicting the hypothesis. Therefore $A^{(r+s) / 2}>B^{(r+s) / 2}$.

Now it is obvious that Corollary 1 and Lemma 11 give the main theorem.

Remark. From Corollary 3, we can write the main theorem in the case $A \geq B$ in a slightly stronger form.

Theorem 2. Suppose that $A, B \in \mathbf{A}$ and $A \geq B, A \not \supset B$. We have two cases:

(1) $A^{r} \geq B^{r}$ for all $r \leq 1$, but $A^{r} \ngtr B^{r}$. This is so iff there exists a $u_{0}$ $\epsilon H^{*}$ such that $F(A) u_{0}=F(B) u_{0}$ for all continuous functions $F$.

(2) If no such $u_{0}$ exists, then $A^{r}>B^{r}$ for all $r<1$ and $A^{r} \underline{\nsupseteq} B^{r}$ for all $r>1$. 
This means that except under a very particular condition, even if we start with a nonstrict inequality $A \geq B$, we can deduce a strict inequality $A^{r}>B^{r}$ for all $r<1$.

\section{REFERENCES}

1. Y. H. Au-Yeung, Some in equalities for the rational powers of a nonnegative definite matrix, Linear Algebra and Appl. 7 (1973), 347-350.

2. F. Riesz and B. Sz.-Nagy, Lecons d'analyse fonctionnelle, Akad. Kiadó, Budapest, 1953; English transl., Functional analysis, Ungar, New York, 1955. MR 14,$286 ; 17,175$.

3. E. Heinz, Beiträge zur Störungstheorie der Spektralzerlegung, Math. Ann. 123 (1951), 415-438. MR 13, 471.

4. T. Kato, Notes on some inequalities for linear operators, Math. Ann. 125 (1952), 208-212. MR 14, 766 .

5. C. Loewner, Über monotone Matrixfunktion en, Math. Z. 38 (1934), 177-216.

6. A. E. Taylor, Introduction to functional analysis, Chapman and Hall, London; Wiley, New York, 1958. MR 20 \#411.

DE PARTMENT OF MATHEMATICS, HONG KONG BAPTIST COLLEGE, KOWLOON, HONG KONG 